\title{
Simultaneous multi-gene mutation screening using SNPscan in patients from ethnic minorities with nonsyndromic hearing-impairment in Northwest China
}

\author{
SHI-HONG DUAN ${ }^{1}$, JIAN-LI MA ${ }^{1}$, XIAO-LONG YANG ${ }^{1}$ and YU-FEN GUO ${ }^{1,2}$ \\ ${ }^{1}$ Department of Otolaryngology-Head and Neck Surgery, Second Hospital of Lanzhou University, \\ Lanzhou, Gansu 730030; ${ }^{2}$ Ministry of Health, Lanzhou, Gansu 730000, P.R. China
}

Received April 22, 2016; Accepted May 11, 2017

DOI: $10.3892 / \mathrm{mmr} .2017 .7431$

\begin{abstract}
The present study aimed to investigate the molecular etiology of nonsyndromic hearing impairment (HI) in hearing impaired populations of Hui, Tibetan, and Tu ethnicities in northwest China. A total of 283 unrelated subjects with HI who attended special education schools in northwest China were enrolled in the present study. Single-nucleotide polymorphisms (SNPs) in three common deafness-related genes, gap junction protein $\beta 2$ (GJB2), solute carrier family 26 member 4 (SLC26A4) and mitochondrially encoded $12 \mathrm{~S}$ RNA (mtDNA12SrRNA), were detected using a SNPscan technique. GJB2 mutations were detected in $14.89 \%$ of Hui patients, $9.37 \%$ of Tibetan patients and $11.83 \%$ of Tu patients. The most prevalent GJB2 mutation in the Hui and Tu patients was c.235delC. In the Tibetan patients, the c.109G>A SNP exhibited the highest allele frequency. SLC26A4 mutations were detected in $10.64 \%$ of Hui patients, $6.25 \%$ of Tibetan patients, and $8.6 \%$ of Tu patients. The most common SLC26A4 mutation was c.919-2A>Gin the Hui, Tibetan, and Tu patients, and the second most common SLC26A4 mutations in these patients were c.1517T $>\mathrm{G}$, c.1226G $>\mathrm{A}$ andc. $2168 \mathrm{~A}>\mathrm{G}$, respectively. The mutation rates ofmtDNA12SrRNA in the Hui, Tibetan, and Tu patients were 1.06, 5.21 , and $5.38 \%$, respectively. These findings demonstrate that the mutation spectra of these deafness-related genes are unique amongst these three ethnic groups. This information will be helpful in designing a protocol for genetic testing for deafness and for achieving accurate molecular diagnoses in northwest China.
\end{abstract}

Correspondence to: Professor Yu-Fen Guo, Department of Otolaryngology-Head and Neck Surgery, Second Hospital of Lanzhou University, 82 Cuiyingmen Street, Lanzhou, Gansu 730030, P.R. China

E-mail: gyflhmm@163.com

Key words: nonsyndromic hearing impairment, GJB2, SLC26A4, mtDNA12SrRNA, minority patients

\section{Introduction}

Hearing impairment (HI) is a genetically heterogeneous disorder in humans, with an incidence of approximately 1:1,000 children worldwide; over half of these cases may be attributed to a genetic cause (1). Nonsyndromic HI (NSHI) accounts for $60-70 \%$ of inherited hearing impairment and involves more than 100 genes with autosomal dominant (20-25\%), autosomal recessive (75-80\%), X-linked (1-2\%) and maternal inheritance $(\geq 1 \%)$ patterns (2). Previous reports conducted in China have suggested that mutations in the gap junction protein $\beta 2$ (GJB2), solute carrier family 26 member 4 (SLC26A4) and mitochondrially encoded 12S RNA (mtDNA12SrRNA) genes are the predominant genetic causes of $\mathrm{HI}$; mutations in these three genes may explain 26.7-35.7\% of the etiology of NSHI (3-5). Mutations in deafness-related genes are currently detected using several different methods, including direct sequencing, microarray analysis, polymerase chain reaction (PCR)-restriction fragment length polymorphism analysis and denatured high-performance liquid chromatography. Although direct sequencing is the gold standard approach for the detection of gene mutations, this strategy is expensive, time-consuming and inefficient for the sequencing of large fragments. The SNPscan technique is high-throughput and cost-effective, and several studies have demonstrated the accuracy, sensitivity and specificity of this technique (6-8). Therefore, SNPscan is considered to be a valid tool for the genetic diagnosis of inherited HI.

Northwest China is home to numerous ethnic groups, including $>40$ ethnic minority groups, some of which are found only in the northwest region, such as the Tu ethnicity. This region is populated by individuals with diverse ethnicities, however, the majority of the residents are of Hui or Tibetan ethnicity. These ethnic minorities reside in separate ethnic neighborhoods and have distinct genetic backgrounds, which are relatively conserved. Therefore, individuals of the Hui, Tibetan and Tu ethnicities residing in this region may present unique mutation spectra of deafness-related genes. The objective of the present study was to investigate the molecular etiology of NSHI using the SNPscan technique, in patients with HI from the Hui, Tibetan and Tu ethnicities residing in northwest China. The information obtained within the present 
study may provide a scientific basis for the diagnosis, intervention and genetic counseling of patients with HI and their families.

\section{Materials and methods}

Patient selection. A total of 283 individuals with NSHI from unrelated families were enrolled; 194 were from Lanzhou Special Education School (Lanzhou, China) and 89 were from Xining Special Education School (Xining, China). An additional 150 region-, age- and ethnicity-matched control individuals with normal hearing were recruited for participation in this study. The study protocol was approved by the Ethics Committee of the Second Hospital of Lanzhou University (Lanzhou, China). Informed consent was obtained from all subjects prior to blood sampling. The medical history of each patient was acquired, and this included age of onset, family history, mother's health during pregnancy, previous history of infection, head trauma and use of aminoglycoside antibiotics. The patients with NSHI received routine physical and otorhinolaryngology examinations. Age-appropriate audiological examinations, including pure-tone audiometry or auditory brainstem response testing, immittance testing, and distortion product otoacoustic emissions testing (9) were performed. All HI subjects demonstrated moderate to profound bilateral sensorineural hearing impairment on audiograms. Patients with middle ear disorders or syndromic HI were excluded from the study. Genomic DNA was extracted from the peripheral blood leukocytes of 283 patients with nonsyndromic hearing impairment and 150 controls with normal hearing using a DNA extraction kit (Axygen; Corning Incorporated, Corning, NY, USA), according to the manufacturer's protocol.

SNPscan for mutation detection. A total of 115 mutations in GJB2, SLC26A4 and mtDNA12SrRNA that were previously identified in $\geq 2$ individuals were selected from a mutation database generated by direct exon-sequencing of the GJB2, mtDNA12SrRNA, and SLC26A4 genes in $>7,000$ patients with HI (10). SNPscan genotyping was performed using a custom-by-design 2x48-plex SNPscan kit (Shanghai Genesky Biotech, Co., Ltd., Shanghai, China). This kit was developed according to a SNP genotyping technology patented by Genesky Biotech, Co., Ltd., based on double ligation and multiplex fluorescence PCR. A ligation mixture was prepared at a volume of $20 \mu \mathrm{l}$ containing $1 \mathrm{X}$ Ligase buffer, $1 \mathrm{X}$ probe mix, $0.5 \mu 1$ ligase and 100-200 ng DNA sample. The ligation reaction was performed using an ABI 2720 thermal cycler (Applied Biosystems; Thermo Fisher Scientific, Inc., Waltham, MA, USA) under the following cycling conditions: $2 \mathrm{~min}$ at $98^{\circ} \mathrm{C}$, followed by 5 cycles of $1 \mathrm{~min}$ at $94^{\circ} \mathrm{C}, 3 \mathrm{~h}$ at $60^{\circ} \mathrm{C}, 2 \mathrm{~min}$ at $94^{\circ} \mathrm{C}$, hold at $58^{\circ} \mathrm{C}$ and immediately stopped with the addition of $20 \mu \mathrm{l} 20 \mathrm{mM}$ EDTA. Multiplex fluorescence PCR reactions were performed for each ligation product. Each PCR mixture was prepared at a volume of $20 \mu$ l containing, 1X PCR buffer, $1 \mu \mathrm{l}$ primer mix, $0.3 \mu \mathrm{l}$ heat activated Taq DNA polymerase and $1 \mu 1$ ligation product. The PCR cycling conditions were as follows: 2 min at $95^{\circ} \mathrm{C} ; 9$ cycles of $20 \mathrm{sec}$ at $94^{\circ} \mathrm{C}, 40 \mathrm{sec}$ cycle at $63-0.5^{\circ} \mathrm{C}$ and $1.5 \mathrm{~min}$ at $72^{\circ} \mathrm{C} ; 25$ cycles of $20 \mathrm{sec}$ at $94^{\circ} \mathrm{C}$, $40 \mathrm{sec}$ at $60^{\circ} \mathrm{C}$, and $1.5 \mathrm{~min}$ at $72^{\circ} \mathrm{C} ; 1 \mathrm{~h}$ at $68^{\circ} \mathrm{C}$; and hold at $4^{\circ} \mathrm{C}$. PCR amplification with blue and red fluorescent dye modified universal primers were performed. The red fluorescence dye was acridine orange and the blue fluorescence dye was 4',6-diamidino-2-phenylindole (both from Shanghai Genesky Biotech, Co., Ltd.). PCR products were separated and detected by capillary electrophoresis on an ABI 3130XL sequencer (Applied Biosystems; Thermo Fisher Scientific, Inc.). Raw data were analyzed using GeneMapper v4.0 (Applied Biosystems; Thermo Fisher Scientific, Inc.). The genotypes at each locus were determined based on the color of the dye label and the fragment sizes of the allele-specific ligation PCR products. For quality control, the assay was randomly repeated for $4 \%$ of the samples and concordant results were obtained. All SNPs were genotyped successfully with a call rate of $>98 \%$.

Statistical analysis. Statistical analysis was performed using SPSS 17.0 software (SPSS, Inc., Chicago, IL, USA). Intergroup differences in rate or frequency were compared using the two-tailed $\chi^{2}$ test. $\mathrm{P}<0.05$ was considered to indicate a statistically significant difference.

\section{Results}

Results overview. The patient cohort consisted of 94 Hui [age, 12.0 \pm 3.1 (range 3-35) years; female/male ratio, 54/40], 96 Tibetan [age, $12.4 \pm 2.8$ (range 8 - 30 ) years; female/male ratio, 45/51] and $93 \mathrm{Tu}$ [age, 13.8 \pm 3.2 (range 4-25) years; female/male ratio, 44/49] individuals. A total of 32/283 patients had a family history of HI (16 Hui, 12 Tibetan and 4 Tu individuals). The remaining 251 patients were diagnosed with sporadic HI. For the control group, 150 individuals with normal hearing from the same region as the case group were recruited, including Hui $(n=50)$, Tibetan $(n=50)$ and Tu $(n=50)$ individuals. The mutation detection rates of the three deafness-related genes in the three ethnicities are presented in Table I. A representative section of the SNPscan results are depicted in Figs. 1-3. DNA samples with the mtDNA12SrRNA m.1494C $>$ T mutation were detected by the red dye labeled probe set in the assay; the wild-type is presented in Fig. 1. DNA samples heterozygous for the $235 \mathrm{delC}$ mutation in GJB2 were detected by the blue dye labeled probe set in the assay; the wild-type is presented in Fig. 2. DNA samples homozygous for the $919-2 \mathrm{~A}>\mathrm{G}$ mutation in SLC26A4 were detected by the blue dye labeled probe set in the assay; the wild-type is presented in Fig. 3.

GJB2. A total of $6 \mathrm{GJB} 2$ variants were identified in the patient cohort (Table II), of which5 were pathogenic, including c.299_300delAT, c.235delC, c.35delG, c.176_191del16 and c. $257 \mathrm{C}>\mathrm{G}$. The c.109G $>$ A SNP was a nucleotide change of unknown significance. The detection rate of GJB2 mutations in the Hui patients was $14.89 \%$ (14/94), and five variants were observed, these were: c.235delC, c.299_300delAT, c.109G >A, c.35delG and c.176_191del16, with allele frequencies of $6.91 \%$ (13/188), $2.66 \%$ (5/188), $2.13 \%$ (4/188), $0.53 \%$ (1/188) and $0.53 \%$ (1/188), respectively. The c.235delC SNP was the most prevalent mutation, accounting for $54.17 \%$ of all mutant alleles. The detection rate of GJB2 mutations was 9.37\% (9/96) in the Tibetan patients, and four variants were detected, these were: c.109G $>$ A, c.235delC, c.299_300delAT and c.257C $>$ G, with allele frequencies of $2.6 \%$ (5/192), $2.08 \%$ (4/192), $0.52 \%$ $(1 / 192)$ and $0.52 \%$ (1/192), respectively. The c.109G $>$ A SNP 
Table I. The detection rates of GJB2, SLC26A4 and mtDNA12SrRNA mutations in 283 Hui, Tibetan and Tu patients with nonsyndromic hearing impairment.

\begin{tabular}{|c|c|c|c|c|c|c|}
\hline \multirow{2}{*}{$\frac{\text { Gene }}{\text { Ethnicity }}$} & \multicolumn{2}{|c|}{ GJB2 } & \multicolumn{2}{|c|}{ SLC26A4 } & \multicolumn{2}{|c|}{ mtDNA12SrRNA } \\
\hline & No. of mutations & Rate (\%) & No. of mutations & Rate (\%) & No. of mutations & Rate (\%) \\
\hline Hui & 14 & 14.89 & 10 & 10.64 & 1 & 1.06 \\
\hline Tibetan & 9 & 9.37 & 6 & 6.25 & 5 & 5.21 \\
\hline $\mathrm{Tu}$ & 11 & 11.83 & 8 & 8.6 & 5 & 5.38 \\
\hline Total & 34 & 12.01 & 24 & 8.48 & 11 & 3.89 \\
\hline
\end{tabular}

GJB2, gap junction protein $\beta 2$; SLC26A4, solute carrier family 26 member 4;mtDNA12SrRNA, mitochondrially encoded 12 S RNA.
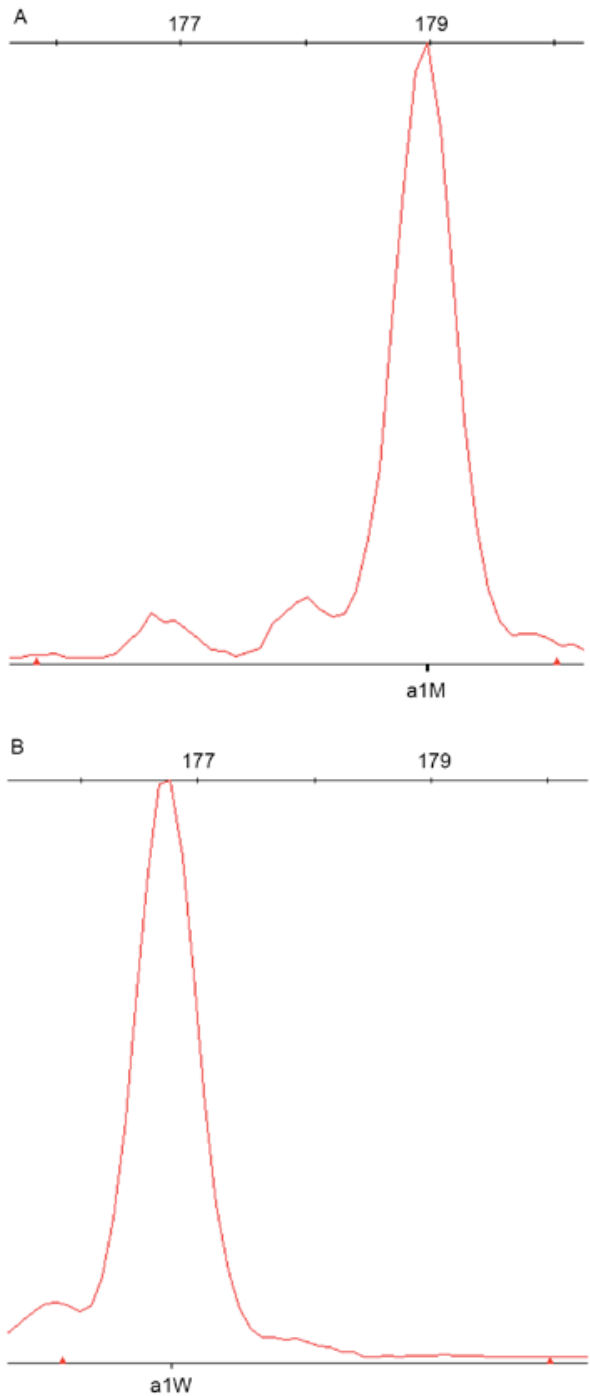

Figure 1. The m.1494C>T SNP in mitochondrially encoded 12S RNA. Homozygous mutant and wild-type sequence variations are presented. (A) The m.1494C $>$ T homozygous mutation in the 179 bp DNA fragment was detected by the red dye labeled probe in the patient sample. (B) The wild-type sequence in the 177th bp of the DNA fragment was detected by the red dye labeled probe in the healthy control. SNP, single-nucleotide polymorphism; M, mutant SNP; W, wild-type SNP.

exhibited the highest allele frequency, accounting for $45.45 \%$ of all mutant alleles. The detection rate of GJB2 mutations
A

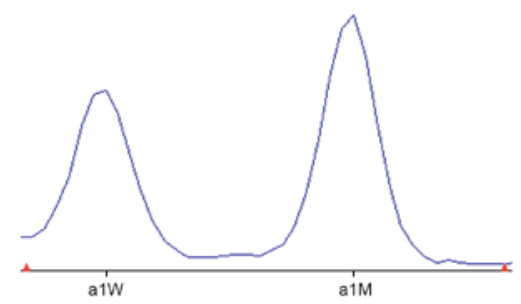

B 103

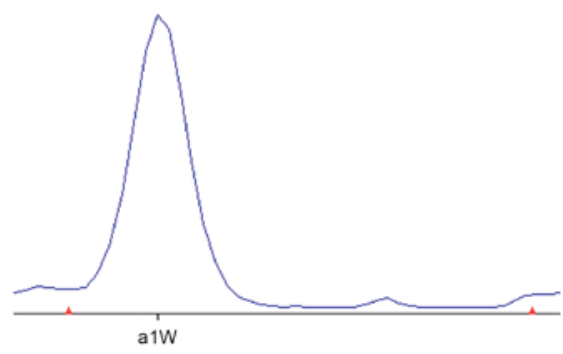

Figure 2. The 235delC SNP in gap junction protein $\beta 2$. Heterozygous and wild-type homozygous sequence variations are presented. (A) The $235 \mathrm{delC}$ heterozygous mutation in the 100 and 102 bp DNA fragment was detected by the blue dye labeled probein the patient sample. (B) The wild-type sequence in the 100th bp of the DNA fragment was detected by the blue dye labeled probe in the healthy control. SNP, single-nucleotide polymorphism; M, mutant SNP; W, wild-type SNP.

was $11.83 \%$ (11/93) in the Tu patients, and three variants were observed, these were: c.235delC, c.109G $>$ A and c.176_191del16, 


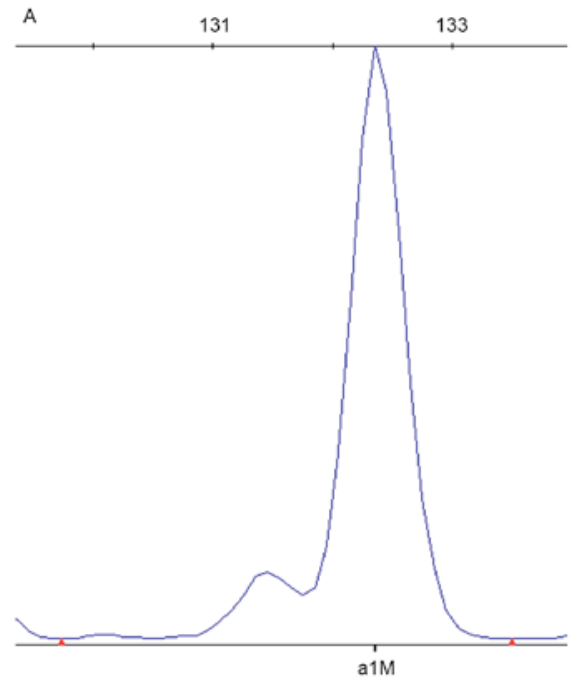

B

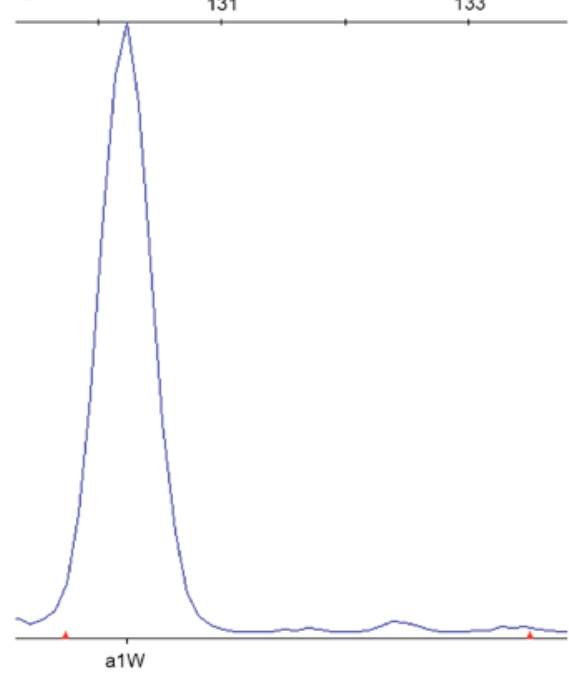

Figure 3. The 919-2A>G SNP in solute carrier family 26 member 4. Homozygous mutant and wild-type sequence variations are presented. (A) The $919-2 \mathrm{~A}>\mathrm{G}$ homozygous mutation in the $132 \mathrm{bp}$ DNA fragment was detected by the blue dye labeled probe in the patient sample. (B) The wild-type sequence in the 130th bp of the DNA fragment was detected by the blue dye labeled probe in the healthy control. SNP, single-nucleotide polymorphism; M, mutant SNP; W, wild-type SNP.

with allele frequencies of $8.06 \%(15 / 186), 1.07 \%(2 / 186)$ and $0.54 \%$ (1/186), respectively. The c.235delC SNP was the most prevalent mutation, accounting for $83.33 \%$ of all mutant alleles.

No significant differences in the detection rate of GJB2 mutations were found amongst the three ethnic groups $(\mathrm{P}>0.05)$. A significant difference in the allele frequency of the c.235delC mutation was observed between the Hui and Tibetan patients $\left(\chi^{2}=5.189, \mathrm{P}<0.05\right)$, and between the Tu and Tibetan patients $\left(\chi^{2}=7.08, \mathrm{P}<0.05\right)$, however, no significant difference in this frequency was detected between the Hui and $\mathrm{Tu}$ patients $\left(\chi^{2}=0.178, \mathrm{P}>0.05\right)$. In the control group, four individuals $(2.67 \%, 4 / 150)$ were revealed to be heterozygous carriers of GJB2 mutations, including one with c. $235 \mathrm{delC}$, one with c.299_300delAT, and two with c.109G>A.

SLC26A4. A total of 7 mutations were identified in this cohort, including c.919-2A $>$ G, c. $2168 \mathrm{~A}>\mathrm{G}$, c.1226G $>\mathrm{A}$, c.754T $>\mathrm{C}$,
c.1520delT, c.1517T $>$ G and c. $249 \mathrm{G}>\mathrm{A}$, (Table III), The detection rate of SLC26A4 mutations in the Hui patients was $10.64 \%$ (10/94), the identified SNPs included c.919-2A $>$ G, c. $2168 \mathrm{~A}>\mathrm{G}, \mathrm{c} .1520 \mathrm{delT}$, c. $1517 \mathrm{~T}>\mathrm{G}$ and c. $249 \mathrm{G}>\mathrm{A}$. The allele frequency of c.919-2A $>\mathrm{G}$ was the highest at $4.79 \%(9 / 188)$, followed by c. $1517 \mathrm{~T}>\mathrm{G}$ at $2.13 \%(4 / 188), \mathrm{c} .249 \mathrm{G}>\mathrm{A}$ at $1.06 \%$ $(2 / 188)$, c. $2168 \mathrm{~A}>\mathrm{G}$ at $0.53 \%(1 / 188)$ and c. $1520 \mathrm{delT}$ at $0.53 \%$ (1/188). Thec.919-2A $>\mathrm{G}$ and $\mathrm{c} .1517 \mathrm{~T}>\mathrm{G}$ mutations were the most prevalent in the Hui patients, accounting for $52.94 \%$ and $23.53 \%$ of all mutant alleles, respectively. The detection rate of SLC26A4 mutations was 6.25\% (6/96) in the Tibetan patients. The identified SNPs included c.919-2A>G, c.2168A $>$ G and c. $1226 \mathrm{G}>\mathrm{A}$. The allele frequency of $\mathrm{c} .919-2 \mathrm{~A}>\mathrm{G}$ was the highest at $3.12 \%(6 / 192)$, followed by c.1226G $>\mathrm{A}$ at $1.04 \%$ $(2 / 192)$ and c. $2168 \mathrm{~A}>\mathrm{Gat} 0.52 \%$ (1/192). The c.919-2A $>\mathrm{G}$ and c. $1226 \mathrm{G}>\mathrm{A}$ mutations were the most prevalent in the Tibetan patients, accounting for $66.67 \%$ and $22.22 \%$ of all mutant alleles, respectively. The detection rate of SLC26A4 mutations was $8.6 \%(8 / 93)$ in the Tu patients. The identified SNPs included c.919-2A $>$ G, c. $2168 \mathrm{~A}>\mathrm{G}$ and c.754T $>$ C. The allele frequency of c.919-2A $>\mathrm{G}$ was the highest at $6.45 \%(12 / 186)$, followed by c. $2168 \mathrm{~A}>\mathrm{G}$ at $1.07 \%(2 / 186)$ and c.754T $>\mathrm{C}$ at $0.54 \%(1 / 186)$.The c.919-2A $>\mathrm{G}$ and c. $2168 \mathrm{~A}>\mathrm{G}$ mutations were the most prevalent in the Tu patients, accounting for $80 \%$ and $13.33 \%$ of all mutant alleles, respectively.

No significant differences in the detection rate of SLC26A4 mutations were found amongst the three ethnic groups $(\mathrm{P}>0.05)$. Furthermore, no significant differences in the allele frequency of the c.919-2A $>$ G mutation were found amongst the three ethnic groups $(\mathrm{P}>0.05)$. In the control group, two individuals $(1.33 \%, 2 / 150)$ were heterozygous for the c.919-2A $>\mathrm{G}$ mutation.

mtDNA12SrRNA. Three Tibetan patients $(3.12 \%, 3 / 96)$ carried the $\mathrm{m} .1555 \mathrm{~A}>\mathrm{G}$ mutation, and two $(2.08 \%, 2 / 96)$ possessed the m.1494C $>$ T mutation. Five Tu patients $(5.38 \%, 5 / 93)$ and one Hui patient $(1.06 \%, 1 / 94)$ carried the $\mathrm{m} .1555 \mathrm{~A}>\mathrm{G}$ mutation. A total of $10 / 11$ patients with the m.1555A $>$ G or the m.1494C $>\mathrm{T}$ mutations had a history of aminoglycoside use. All of the mutations detected in mtDNA12SrRNA were in a homoplasmic state. No significant differences in the mutation rates of mtDNA12SrRNA were observed amongst the three ethnic groups $(\mathrm{P}>0.05)$. None of the 150 control individuals were found to carry the m.1555A $>$ G mutation or the m.1494C $>$ T mutation.

\section{Discussion}

The present study performed mutation analysis using the SNPscan technique to evaluate 283 patients with moderate to profound sensorineural NSHI. The detection rates of GJB2 mutations were $14.89,9.37$ and $11.83 \%$ in the Hui, Tibetan and $\mathrm{Tu}$ patients, respectively. The detection rates of SLC26A4 mutations were 10.64, 6.25 and $8.6 \%$ in the Hui, Tibetan and Tu patients, respectively. The mutations rates of mtDNA12SrRNA in the Hui, Tibetan and Tu patients were $1.06,5.21$ and $5.38 \%$, respectively. No significant differences in the detection rates of GJB2, SLC26A4 and mtDNA12SrRNA mutations were found amongst the three ethnic groups. Notably, the mutation spectra of the common 
Table II. Gap junction protein $\beta 2$ genotypes of 283 minority patients with nonsyndromic hearing impairment.

\begin{tabular}{lccc}
\hline Genotype & Hui patients $(\mathrm{n})$ & Tibetan patients $(\mathrm{n})$ & Tu patients $(\mathrm{n})$ \\
\hline 235delC/235delC & 5 & 1 & 6 \\
299_300delAT/299_300delAT & 2 & 0 & 0 \\
235delC/299_300delAT & 1 & 0 & 0 \\
35delG/235delC & 1 & 0 & 0 \\
176_191del16/235delC & 1 & 0 & 1 \\
257C>G/299_300delAT & 0 & 1 & 0 \\
235delC/wt & 0 & 2 & 2 \\
109G>A/wt & 4 & 5 & 2 \\
Total & 14 & 9 & 11 \\
\hline
\end{tabular}

WT, wild-type.

Table III. Solute carrier family 26 member 4 genotypes of 283 minority patients with nonsyndromic hearing impairment.

\begin{tabular}{lccc}
\hline Genotype & Hui patients $(\mathrm{n})$ & Tibetan patients $(\mathrm{n})$ & Tu patients $(\mathrm{n})$ \\
\hline $919-2 \mathrm{~A}>\mathrm{G} / 919-2 \mathrm{~A}>\mathrm{G}$ & 3 & 1 & 5 \\
$919-2 \mathrm{~A}>\mathrm{G} / 2168 \mathrm{~A}>\mathrm{G}$ & 0 & 1 & 1 \\
$754 \mathrm{~T}>\mathrm{C} / 919-2 \mathrm{~A}>\mathrm{G}$ & 0 & 0 & 1 \\
$1226 \mathrm{G}>\mathrm{A} / 1226 \mathrm{G}>\mathrm{A}$ & 0 & 1 & 0 \\
$1520 \mathrm{delT} / 2168 \mathrm{~A}>\mathrm{G}$ & 1 & 0 & 0 \\
$1517 \mathrm{~T}>\mathrm{G} / 1517 \mathrm{~T}>\mathrm{G}$ & 2 & 0 & 0 \\
$249 \mathrm{G}>\mathrm{A} / 249 \mathrm{G}>\mathrm{A}$ & 1 & 0 & 0 \\
$919-2 \mathrm{~A}>\mathrm{G} / \mathrm{wt}$ & 3 & 3 & 0 \\
$2168 \mathrm{~A}>\mathrm{G} / \mathrm{wt}$ & 0 & 0 & 8 \\
Total & 10 & 6 & 8 \\
\hline
\end{tabular}

WT, wild-type.

deafness-related genes differed amongst the three ethnic groups.

Previous reports have suggested that mutations in GJB2 are the most common causes of NSHI in several populations $(11,12)$. The prevalence of GJB2 mutations appears to vary amongst different ethnic groups. The results of this study indicated that the detection rates of GJB2 mutations in the Hui, Tibetan and Tu patients were 14.89, 9.37, and $11.83 \%$, respectively. However, Dai et al (13) reported that the detection rate of GJB2 mutations was 19.1\% (313/1640) in Han Chinese NSHI patients from different regions of China. A significant difference in this rate was observed between the Tibetan patients and the aforementioned Han Chinese patients $\left(\chi^{2}=5.66, \mathrm{P}<0.05\right)$, consistent with the report of Dai et al (13). No significant differences were discovered between the Han Chinese group and Hui and Tu ethnic groups in the present study $(\mathrm{P}>0.05)$. The mutation spectra of GJB2 are distinct amongst HI populations of different ethnicities. Numerous studies have demonstrated that c. $35 \mathrm{delG}$, c.167delT and c. $235 \mathrm{delC}$ are the most prevalent mutations in Caucasians, Ashkenazi Jews and a pan-Asian cohort, respectively (14). In the present study, c.235delC was the most prevalent mutation in Hui and Tu patients. This finding is consistent with the observation that the c.235delC mutation is the most common mutation in Eastern Asians (15). Furthermore, the allele frequency of c.235delC was lower in the Tibetan patients compared with the Hui and Tu patients, in agreement with the report of Dai et al (15). The c.109G>A mutation exhibited the highest allele frequency in the Tibetan patients. This mutation is common in East Asians, with an allele frequency of $6.7 \%(185 / 2744)$ in Han Chinese patients with NSHI (13). No statistical difference in this frequency was observed between the Tibetan patients and the aforementioned Han Chinese patients $\left(\chi^{2}=2.275, \mathrm{P}>0.05\right)$. The pathogenicity of the c. $109 \mathrm{G}>\mathrm{A}$ variant is controversial; recent research has revealed that it is associated with mild to moderate hearing impairment (16-17). In the present study, the allele frequency of c.109G >A was $2.6 \%$ (5/192) in the Tibetan patients and $2 \%$ $(1 / 50)$ in the Tibetan control subjects; no significant difference in this frequency was found between the Tibetan patient and control groups $\left(\chi^{2}=0.06, \mathrm{P}>0.05\right)$.

Mutations in SLC26A4 are responsible for both syndromic and nonsyndromic hearing impairment; they are the second most common genetic cause of NSHI in China (5). The prevalence of SLC26A4 mutations varies amongst different ethnic 
groups. In the present study, the detection rates of SLC26A4 mutations were 10.64, 6.25 and $8.6 \%$ in the Hui, Tibetan and Tu patients, respectively. Yuan et al (18) reported that the detection rate of SLC26A mutations was $16.55 \%(315 / 1903)$ in Han Chinese NSHI patients from different regions of China. A significant difference in this rate was found between the Han Chinese patients and Tibetan patients $\left(\chi^{2}=7.197, \mathrm{P}<0.05\right)$, this finding is consistent with a report by Yuan et al (18) that the detection rate of SLC26A4 mutations in the Tibetan HI population was significantly lower compared with the Han Chinese population. No significant differences were observed between the aforementioned Han Chinese group and Hui and Tu ethnic groupsin the present study $(\mathrm{P}>0.05)$. The mutation spectra of SLC26A4 varied amongst different ethnic groups. Previous studies have indicated that the most common mutations in the SLC26A4 gene are p.T416P and IVS8+1G>A in northern European populations and c.2168A $>\mathrm{G}$ in Japanese or Korean populations (19-21), whereas the most common SLC26A4 mutations are c.919-2A $>$ G and c.2168A>G in Han Chinese populations $(22,23)$. In the present study, it was observed that different ethnicities demonstrated distinct SLC26A4 mutation spectra. The c.919-2A>G was the most common mutation in the Hui, Tibetan and $\mathrm{Tu}$ patients, however, c. $1517 \mathrm{~T}>\mathrm{G}, \mathrm{c} .1226 \mathrm{G}>\mathrm{A}$ and c.2168A $>\mathrm{G}$ were the second most common mutations in these patients, respectively. These results suggest that ethnic background is a significant factor contributing to differences in the SLC26A4 mutation spectra amongst these ethnicities.

The A1555 G and C1494T mutations in mtDNA12SrRNA have been associated with aminoglycoside-induced deafness and nonsyndromic hearing impairment in several families of different ethnicities (24-27). In particular, the A1555 G mutation is the most commonly detected, and its prevalence fluctuates with ethnic variation (28). Compared with the A1555 G mutation, the C1494T mutation exhibits a lower carrier frequency in Chinese patients with NSHI (29). Published reports have indicated that the incidence of the m.1555A $>\mathrm{G}$ mutation is higher in Asian HI populations compared with Caucasian populations (30). In the present study, the mutation rates of $\mathrm{m} .1555 \mathrm{~A}>\mathrm{G}$ were $3.12,5.38$ and $1.06 \%$ in the Hui, Tibetan and Tu patients, respectively. Guo et al (31) reported that the mutation rate of $\mathrm{m} .1555 \mathrm{~A}>\mathrm{G}$ was $6.09 \%$ (113/1856) in Han Chinese NSHI patients in northwest China. No significant differences in this rate were observed between the aforementioned Han Chinese group and Tibetan and Tu ethnic groups in the present study $(\mathrm{P}>0.05)$. However, significant differences were observed between the Han Chinese patients and Hui patients $\left(\chi^{2}=4.103, \mathrm{P}<0.05\right)$. The differences in the m.1555A $>\mathrm{G}$ mutation rate may be due to geographical and environmental differences. Given that ethnic background influences the spectrum of gene mutations present, the western Eurasian-specific haplogroup frequency of mtDNAs in the Hui population was 6.7\%; however, no western Eurasian type was found in Han samples from the same region (32). Furthermore, the A1555 G and C1494T mutations in the highly conserved coding site of mtDNA12SrRNA are the predominant ototoxic targets of aminoglycoside antibiotics, especially the A1555 G mutation. These mutations increase sensitivity to aminoglycoside ototoxicity, thus leading to these ototoxic effects (33). In the present study, 10/11 patients with A1555 G or C1494T mutations had a history of aminoglycoside use. One Tibetan patient without a history of aminoglycoside exposure carried the C1494T mutation and exhibited late-onset deafness. Therefore, it may be hypothesized that active genetic counseling and intervention combined with avoidance of aminoglycoside use in patients with HI, and their matrilineal relatives, may limit the occurrence of HI.

The present study demonstrated that mutation screening using the SNPscan assay is a powerful and effective method for the evaluation of a large deaf cohort. However, the sample size of the present study was too small to allow for final conclusions to be made, and more patients with nonsyndromic hearing impairment will be recruited for future studies. A total of $24.38 \%$ of the patients with HI demonstrated evidence of genetic involvement; $12.01,8.48$ and $3.89 \%$ of the patients demonstrated inherited hearing impairment caused by GJB2, SLC26A4 and mtDNA12SrRNA mutations, respectively. Furthermore, the mutation spectra of the common deafness-related genes differed amongst HI populations of the Hui, Tibetan and Tu ethnicities. The information reported in this study will be helpful in designing a protocol for genetic testing for $\mathrm{HI}$, and for achieving accurate molecular diagnoses in northwest China.

\section{Acknowledgements}

The present study was supported by the National Natural Science Foundation of China (grant no. 81172765).

\section{References}

1. Morton NE: Genetic epidemiology of hearing impairment. Ann N Y Acad Sci 630: 16-31, 1991.

2. Bitner-Glindzicz M: Hereditary deafness and phenotyping inhumans. Br Med Bull 63: 73-94, 2002.

3. Guo YF, Liu XW, Guan J, Han MK, Wang DY, Zhao YL, Rao SQ and Wang QJ: GJB2, SLC26A4 and mitochondrial DNA A $1555 \mathrm{G}$ mutations in prelingual deafness in Northern Chinese subjects. Acta Otolaryngol 128: 297-303, 2008.

4. Yuan Y, You Y, Huang D, Cui J, Wang Y, Wang Q, Yu F, Kang D, Yuan H, Han D and Dai P: Comprehensive molecular etiology analysis of nonsyndromic hearing impairment from typical areas in China. J Transl Med 7: 79, 2009.

5. Xin F, Yuan Y, Deng X, Han M, Wang G, Zhao J, Gao X, Liu J, Yu F, Han D and Dai P: Genetic mutations in nonsyndromic deafness patients of Chinese minority and Han ethnicities in Yunnan, China. J Transl Med 11: 312, 2013

6. Chen X, Li S, Yang Y, Yang X, Liu Y, Liu Y, Hu W, Jin L and Wang X: Genome-wide association study validation identifies novel loci for atherosclerotic cardiovascular disease. J Thromb Haemost 10: 1508-1514, 2012.

7. Tang LL, Chen FY, Wang H, Hu XL, Dai X, Mao J, Shen ZT, Wu YH, Wang SM, Hai J, et al: Haplotype analysis of eight genes of the monoubiquitinated FANCD2-DNA damage-repair pathway in breast cancer patients. Cancer Epidemiol 37: 311-317, 2013.

8. Yin J, Wang L, Shi Y, Shao A, Tang W, Wang X, Ding G Liu C, Chen S and Gu H: Interleukin 17A rs4711998 A>G polymorphism was associated with a decreasedrisk of esophageal cancer in a Chinese population. Dis Esophagus 27: 87-92, 2014.

9. Erenberg A, Lemons J, Sia C, Trunkel D and Ziring P: Newborn and infant hearing loss: Detection and intervention. American Academy of Pediatrics. Task Force on Newborn and Infant Hearing, 1998-1999. Pediatrics 103: 527-530, 1999.

10. Du W, Cheng J, Ding H, Jiang Z, Guo Y and Yuan H: A rapid method for simultaneous multi-gene mutation screening in children with nonsyndromic hearing loss. Genomics 104: 264-270, 2014 
11. Wilcox SA, Saunders K, Osborn AH, Arnold A, Wunderlich J, Kelly T, Collins V, Wilcox LJ, McKinlay Gardner RJ, Kamarinos M, et al: High frequency hearing loss correlated with mutations in the GJB2 gene. Hum Genet 106: 399-405, 2000.

12. Estivill X, Fortina P, Surrey S, Rabionet R, Melchionda S, D'Agruma L, Mansfield E, Rappaport E, Govea N, Milà M, et al: Connexin-26 mutations in sporadic and inherited sensorineural deafness. Lancet 351: 394-398, 1998.

13. Dai P, Yu F, Han B, Liu X, Wang G, Li Q, Yuan Y, Liu X, Huang D, Kang D, et al: GJB2 mutation spectrum in 2,063 Chinese patient with nonsyndromic hearing impairment. J Transl Med 7: 26, 2009.

14. Kenneson A, Van Naarden Braun K and Boyle C: GJB2 (connexin 26) variants and nonsyndromic sensorineural hearing loss: A HuGE review. Genet Med 4: 258-274, 2002.

15. Dai P, Yu F, Han B, Yuan Y, Li Q, Wang G, Liu X, He J, Huang D, Kang D, et al: The prevalence of the 235 delC GJB2 mutation in a Chinese deaf population. Genet Med 9: 283-289, 2007.

16. Li L, Lu J, Tao Z, Huang Q, Chai Y, Li X, Huang Z, Li Y, Xiang M, Yang J, et al: The p.V37I exclusive genotype of GJB2: A genetic risk indicator of postnatal permanent childhood hearing impairment. PLoS One 7: e36621, 2012.

17. Gallant E, Francey L, Tsai EA, Berman M, Zhao Y, Fetting H, Kaur M, Deardorff MA, Wilkens A, Clark D, et al: Homozygosity for the V37I GJB2 mutation in fifteen probands with Mild to Moderate sensorineural hearing impairment: Further confirmation of pathogenicity and haplotype analysis inAsian populations. Am J Med Genet 161A: 2148-2157, 2013.

18. Yuan Y, Guo W, Tang J, Zhang G, Wang G, Han M, Zhang X, Yang S, He DZ and Dai P: Molecular epidemiology and functional assessment of novel allelic variants of SLC26A4 in nonsyndromic hearing loss patients with enlarged vestibular aqueduct in China. PLoS One 7: e49984, 2012.

19. Campbell C, Cucci RA, Prasad S, Green GE, Edeal JB, Galer CE, Karniski LP, Sheffield VC and Smith RJ: Pendred syndrome, DFNB4, and PDS/SLC26A4 identification of eight novel mutations and possible genotype-phenotype correlations. Hum Mutat 17: 403-411, 2001.

20. Tsukamoto K, Suzuki H, Harada D, Namba A, Abe S and Usami S: Distribution and frequencies of PDS (SLC26A4) mutations in Pendred syndrome and nonsyndromic hearing loss associated with enlarged vestibular aqueduct: A unique spectrum of mutations in Japanese. Eur J Hum Genet 11: 916-922, 2003.

21. Park HJ, Shaukat S, Liu XZ, Hahn SH, Naz S, Ghosh M, Kim HN, Moon SK, Abe S, Tukamoto K, et al: Origins and frequencies of SLC26A4 (PDS) mutations in east and south Asians: Global implications for the epidemiology of deafness. J Med Genet 40: 242-248, 2003

22. Wang QJ, Zhao YL, Rao SQ, Guo YF, Yuan H, Zong L, Guan J, $\mathrm{Xu}$ BC, Wang DY, Han MK, et al: A distinct spectrum of SLC26A4 mutations in patients with enlarged vestibular aqueduct in China. Clin Genet 72: 245-254, 2007.
23. Dai P, Li Q, Huang D, Yuan Y, Kang D, Miller DT, Shao H, Zhu Q, He J, Yu F, et al: SLC26A4 c.919-2A>G varies among Chinese ethnic groups as a cause of hearing loss. Genet Med 10: 586-592, 2008

24. Jacobs HT, Hutchin TP, Käppi T, Gillies G, Minkkinen K, Walker J, Thompson K, Rovio AT, Carella M, Melchionda S, et al: Mitochondrial DNA mutations in patients with postlingual, nonsyndromic hearing impairment. Eur J Hum Genet 13: 26-33, 2005.

25. Li R, Xing G, Yan M, Cao X, Liu XZ, Bu X and Guan MX: Cosegregation of C-insertion at position 961 with A1555G mutation of mitochondrial 12S rRNA gene in a large Chinese family with maternally inherited hearing loss. Am J Med Genet A 124A: 113-117, 2004

26. Zhao H, Young WY, Yan Q, Li R, Cao J, Wang Q, Li X, Peters JL, Han D and Guan MX: Functional characterization of the mitochondrial 12S rRNA C1494T mutation associated with aminoglycoside-induced and non-syndromic hearing loss. Nucleic Acids Res 33: 1132-1139, 2005.

27. Yuan HJ, Chen J, Liu X, Cheng J, Wang X, Yang L, Yang S, Cao J, Kang D, Dai P, et al: Coexistence of mitochondrial $12 \mathrm{~S}$ rRNA C1494T and CO1/tRNA(Ser(UCN)) G7444A mutations in two Han Chinese pedigrees with aminoglycoside-induced and non-syndromic hearing loss. Biochem Biophys Res Commun 362: 94-100, 2007.

28. Prezant TR, Agapian JV, Bohlman MC, Bu X, Oztas S, Qiu WQ, Arnos KS, Cortopassi GA, Jaber L, Rotter JI, et al: Mitochondrial ribosomal RNA mutation associated with both antibiotic-induced and non-syndromic deafness. Nat Genet 4: 289-294, 1993.

29. Li Q, Yuan YY, Huang DL, Han DY and Dai P: Rapid screening for the mitochondrial DNA C1- 494T mutation in a deaf population in China using real-time quantitative PCR. Acta Otolaryngol 132: 814-818, 2012.

30. Li R, Greinwald JH Jr, Yang L, Choo DI, Wenstrup RJ and Guan MX: Molecular analysis of the mitochondrial $12 \mathrm{~S}$ rRNA and tRNASer(UCN) genes in paediatric subjects with non-syndromic hearing loss. J Med Genet 41: 615-620, 2004.

31. Guo YF, Liu XW, Xu BC, Zhu YM, Wang YL, Zhao FF, Wang DY, Zhao YL, Ji YB and Wang QJ: Analysis of a large-scale screening of mitochondrial DNA m.1555A $>\mathrm{G}$ mutation in 2417 deaf-mute students in northwest of China. Genet Test Mol Biomarkers 14: 527-531, 2010.

32. Yao YG, Kong QP, Wang CY, Zhu CL and Zhang YP: Different matrilineal contributions to genetic structure of ethnic groupsin the silk road region in China. Mol Biol Evol 21: 2265-2280, 2004.

33. Zhao H, Li R, Wang Q, Yan Q, Deng JH, Han D, Bai Y, Young WY and Guan MX: Maternally inherited aminoglycoside-induced and nonsyndromic deafnessis associated with the novel C1494T mutation in the mitochondrial12S rRNA gene in a large Chinese family. Am J Hum Genet 74: 139-152, 2004. 